\title{
A Preprint Server for Chemistry
}

As the concept of preprint servers for the scientific community in general continues to be debated, $C I$ has asked Bill Town, managing director of ChemWeb.com, to review for us the development and status of the initiative to provide a preprint server for the chemistry community.

\section{by Bill Town}

Before the advent of the Internet, scientists would typically only publish their work as research articles in standard peer-reviewed journals. In this process, an article is submitted to a board of editors and occasionally, the article is accepted in the state in which it was submitted. However, articles are often returned to the authors with a list of corrections that should be made before publication. In the field of chemistry the duration of the entire submission process-from completion of first draft to publication - can often extend to one year or more.

Authors within different scientific communities have for some time discussed their research by exchanging articles-"preprints"-amongst themselves before formal publication. Some authors choose to do this by posting articles to their own Web site. The alternative, which is growing in popularity, is to submit to"preprint servers"-permanent and freely available databases on the Internet. This process does not involve any peer review.

Perhaps the most successful example of a preprint server is the arXiv server, ${ }^{1}$ covering high-energy physics and mathematics. Founded in 1991 by Paul Ginsparg, the arXiv server is now host to more than 300000 preprints with just under 3000 new preprints being submitted every month. Other disciplines have been slower to follow this trend. Recently, similar services have been set-up, including Cogprints ${ }^{2}$ (for psychology and biology) and the Chemical Physics Preprint Database hosted at Brown University. ${ }^{3}$ In August 2000, ChemWeb.com launched the Chemistry Preprint Server (CPS), ${ }^{4}$ which is the first preprint server to cover the entire field of chemistry. The CPS was officially launched at the fall ACS National Meeting on 21 August 2000 in Washington, D.C. ${ }^{5}$ By the launch date, 20 preprints had already been submitted.

Whether a chemistry preprint server will prove to be as successful as the arXiv server is for physics and mathematics is a question that will be answered by chemists themselves. The CPS was set up as an experimental service to examine the response of the community. ChemWeb.com believes that preprint servers have many advantages for researchers. For example, articles submitted to the CPS may take any format from an initial draft to a complete article ready for publication. Authors may continually revise the original version of the article and any number of supporting files may be added. In this way, scientific information is disseminat- ed very rapidly and is archived permanently in electronic format. Furthermore, all preprints submitted to the CPS have their own discussion group where all users may discuss the article online.

Critics have raised concerns regarding the use of preprint servers for the dissemination of chemical information. 6,7 One such concern is the possible poor quality of information hosted online without any formal peer review in place. In other words, how do you extract the signal from the noise?

In general, the CPS received a positive response at the ACS meeting, where there was a plenary presidential event and Webcast entitled "The Impact of Preprint Servers in Scholarly Publishing." 8 The opinions expressed at this event were mixed. Again, the primary concerns were issues relating to prior publication and the possible lack of quality control. This debate has been discussed comprehensively in Science: "Chemists Toy with the Preprint Future." 7

\section{Preprint servers are permanent and freely available databases on the Internet.}

To counter the concerns there are comprehensive searching and browsing facilities available on the CPS which allow the user to rapidly find an article of interest. All articles are screened to ensure that they do genuinely contain scientific content related to chemistry. Authors also must write in a standard format. In addition, the preprint discussion groups could provide an environment where an article is truly reviewed by one's peers. Every article accepted on the CPS is given a unique identifier - this supports the position of the CPS as a permanent Web archive and distribution medium.

Whether peer review really does guarantee the quality of a published article is another question. For printed media, the number of articles being submitted to peer-reviewed journals is growing so rapidly that editors and publishers now face an increasingly difficult task. In principle, they should ensure that the work is original and based on true scientific research. The introduction of preprint servers, as an additional step before formal publication, may in fact aid this process. Further information may be found in the recent article "Chemical E-Prints: The Ostriches" by Ray Dessy, published in Trends in Analytical Chemistry. ${ }^{9}$ 
Perhaps the most important concern at the present time involves the prior-publication policies of all publishers within the field of chemistry. Currently, there is significant confusion over which publishers will publish preprinted articles. For example, Elsevier Science, the Royal Society of Chemistry, Nature, and many physics publishers will accept preprinted articles for publication in their journals. However, the American Chemical Society released a policy statement ${ }^{9}$ stating that articles that have previously been made available on a preprint server will not be considered for publication. This problem arises from the policies of individual publishers and should not be confused with the issue of copyright or prior art issues for patents. For example, all authors must retain the article copyright to submit to the CPS. Furthermore, all preprints are stamped with the time of upload and a unique citation reference.

Politics aside, the CPS has been set up as an experiment in scientific communication for the worldwide chemistry community, and its success will be determined by its acceptance within the community. Although early signs do indicate a positive response, time will tell if researchers in the field of chemistry will adopt the CPS in the same way physicists and mathematicians have the arXiv preprint server.

The CPS was modelled on the arXiv preprint server, described above, to provide the first preprint server to cover the entire field of chemistry. In setting up the service, ChemWeb.com has constantly referred to the Open Archive Initiative ${ }^{1} 1$ (OAI) for e-print archives. The CPS is now a compliant data provider for the OAI. The general purpose of the OAI is to set standards for the transfer of information between different Web servers. In this way, users of remote preprint servers are able to search through all of the information hosted on the CPS.

From the outset, an advisory board was set up for the CPS so that its independent status is maintained. The advisory board also ensures that the server develops and adapts to meet the needs of the chemical community. Some of the community's most respected names have shown their support for the CPS by becoming founder members of the advisory board, including Professor Pieter Steyn, current president of IUPAC. Other members include Professor Peter Atkins, Oxford University; Dr. Steve Bachrach, Internet Journal of Chemistry; Dr. Ad Bax, National Institutes of Health; Professor Ray Dessy, Virginia Tech; Dr. Jonathan Goodman, Cambridge University; Dr. Bill Milne, JCICS; Professor Paul Schleyer, University of Erlangen-Nuremberg; Dr. Edlyn Simmons, The Procter \& Gamble Company; and Dr. Engelbert Zass, ETH Zürich.

Since the CPS was launched in August 2000, almost 500 preprints have been uploaded and made available for browsing, review, and discussion. The topics encompass all areas of chemistry, from computational and physical chemistry to biochemistry. To date, submissions have been made from 51 different countries. The largest num-

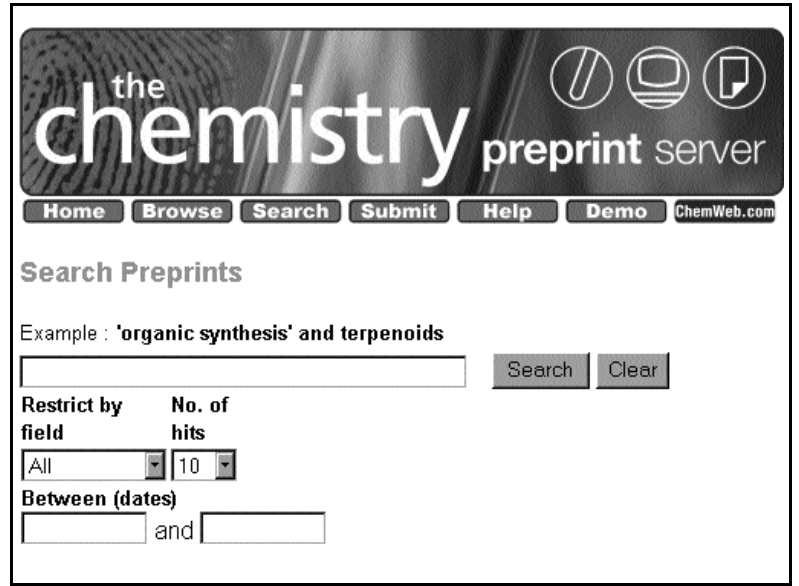

ber have been from the United States, but significant numbers have also come from the United Kingdom, Western and Eastern Europe, Russia, and India.

It is important to note that the CPS is still very much an experiment in scientific communication for the chemistry community. However, usage analysis of the service during its first two years of operation does indicate that it is receiving a very positive response. As the CPS becomes better known within the academic and industrial communities, the number of submitted papers will continue to grow. The CPS will develop and adapt to meet the needs of the chemical community.

\section{References}

1. http://arxiv.org

2 http://cogprints.soton.ac.uk

3 http://www.chem.brown.edu/chem-ph.html

4. http://preprint.chemweb.com

5. http://www.chemweb.com/home/prpages/ ppslive.html

6. Gorman, J. The End of Good Science?. Science News 2001, 159, 76-78

7. Service, R. Chemists Toy With the Preprint Future. Science 2001, 289, 5484, 1445-1446. http://www.sciencemag.org/cgi/content/full/289/54 $84 / 1445 \mathrm{a}$

8. http://pubs.acs.org/cen/preprint.html

9. Dessy, R. Chemical E-prints: The Ostriches. Trends Anal. Chem. 2000, 19, 587-592. http://www.elsevier.nl/homepage/saa/trac/column/preprint.htm

10. http://www.openarchives.org

Bill Town<bill.town@chemweb.com> is managing director of ChemWeb.com, and Titular Member of the IUPAC Chemical Nomenclature and Structure Representation Division.

\section{inii] www.preprint.chemweb.com}

